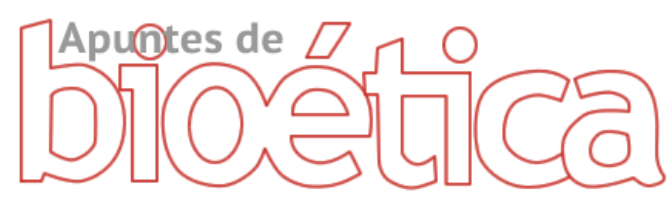

https://doi.org/ 10.35383/apuntes.v3i1.367

\title{
La dignidad del enfermo en situación terminal
}

\author{
Campos Olazabal P. ${ }^{1}$
}

\section{INFORMACIÓN DEL ARTÍCULO RESUMEN}

Historia del artículo:

Recibido el 23 de abril de 2020

Aceptado el 25 de julio de 2020

\section{Palabras claves:}

Dignidad humana

Enfermo en situación terminal

Derechos humanos

Eutanasia

Cuidados paliativos

La dignidad del enfermo en situación terminal debe ser apreciada, por su condición de ser humano, ostentando la correspondiente dignidad por el hecho de "ser", de su existencia. Es conocido que las situaciones terminales cambian las condiciones en las que se encuentra el paciente, pero no es lícito pretender que las circunstancias afecten su dignidad intrínseca, de la misma manera que no puede pretenderse que por encontrarse en una situación terminal no tenga derecho a la vida. Las circunstancias adversas pueden estar presentes en la vida del ser humano, pero siempre será un fin y nunca un medio, en cuyo marco la dignidad del paciente terminal debe ser respetada, debiendo asegurarse las condiciones necesarias de cómo serán brindadas las atenciones correspondientes hasta el final de su vida, más no direccionar acciones tendentes a cuándo poner término a su vida.

\section{The dignity of the sick in terminal situation}

\section{ABSTRACT}

\section{Keywords:}

Human dignity

Terminally ill

Human rights

Euthanasia

Palliative care
The dignity of the terminally ill patient must be appreciated, for the condition of being human, showing the corresponding dignity for the fact of "being", of the existence. It is known that terminal situations change the conditions in which the patient finds himself, but it is not lawful to claim that circumstances affect his intrinsic dignity, in the same way that it cannot be claimed that because he is in a terminal situation he does not have the right to lifetime. Adverse circumstances may be present in the life of the human being, but it will always be an end and never a means, within which the dignity of the terminally ill patient must be respected, and the necessary conditions must be ensured of how the corresponding care will be provided until the end. of your life, but not direct actions aimed at when to end your life.

\footnotetext{
${ }^{1}$ Doctora en Medicina por la Universidad Cayetano Heredia, Maestría en Bioética y Biojurídica por la Universidad Católica Santo Toribio de Mogrovejo. Rectora de la Universidad Católica Santo Toribio de Mogrovejo, Chiclayo, Perú. Email: pcampos@usat.edu.pe. ORCID: https://orcid.org/0000-0002-4380-3570
} 


\section{Dignidad humana y enfermo terminal: aproximaciones conceptuales}

Las definiciones tanto de dignidad humana como del enfermo en situación terminal son de importancia para tratar el tema, y no están exentas de presentar muchas aristas, por la complejidad de sus atributos. Así, diremos con Aparisi Miralles (2013) que el concepto de dignidad humana desde una perspectiva filosófica y religiosa tiene un largo trayecto, pero, desde el punto de vista jurídico, solo se vio reflejada hasta el año 1945 con la Carta de las Naciones Unidas y tres años más tarde con la Declaración Universal de los Derechos Humanos. La Carta de las Naciones Unidas en 1945, considera que "los derechos humanos son inherentes a todos los seres humanos sin distinción (...) estos derechos son interrelacionados, independientes e indivisibles" (Aparisi Miralles, 2013). Posteriormente la Declaración Universal de los Derechos Humanos (1948) establece en su preámbulo: "la libertad, la justicia y la paz en el mundo tienen por base el reconocimiento de la dignidad intrínseca y de los derechos iguales e inalienables de todos los miembros de la familia humana".

A nivel constitucional el principio de la dignidad humana también ha sido reconocido como un principio fundamental y se encuentra contenido en la constitución de muchos países del mundo. Como ejemplo tenemos la Constitución Española (art 10.1) en donde se cita: "La dignidad de la persona, los derechos inviolables que le son inherentes (...) son fundamentos del orden político y de la paz social" (Aparisi Miralles, 2013). En el Perú nuestra Constitución también lo regula en el artículo 2 que a la letra establece: "La defensa de la persona humana y el respeto de su dignidad son el fin supremo de la sociedad y del estado"; como se aprecia en el Perú, el principio de la dignidad humana ha sido elevado a rango constitucional y a finalidad del Estado.
Al acercarnos a la definición del principio de la dignidad humana notamos que es difícil encontrar una definición acabada; así se puede decir con Spaemann (1988) que dignidad es un término de complejo entendimiento conceptual. Si nos remontamos a las diversas Declaraciones ya citadas admitiremos que la dignidad humana es una dimensión intrínseca del ser humano y que posee un carácter ontológico independiente de cualquier sentido religioso.

Para la filosofía antigua la dignidad humana es un presupuesto, la dignitates significaba en el medioevo lo mismo que los axiomas para los griegos, proposiciones evidentes por sí mismas e indemostrables como lo cita Ballesteros. Tomás de Aquino, en el capítulo 1 de la Suma Teológica, proponía que "el término dignidad es algo absoluto y pertenece a la esencia". D'Agostino (2002) señala, por ejemplo, "es un hecho que en nuestro tiempo actúa una conciencia colectiva que percibe que la subjetividad humana no puede ser cosificada, porque ser sujetos lleva consigo una identidad que no admite equivalentes funcionales". De esta manera el valor que todo ser humano tiene en sí mismo es independiente de cualquier otro factor, lo cual lo hace merecedor de un respeto incondicionado como vuelve a subrayar (Spaemann, 1988).

Por su parte García Cuadrado (2012) lo resume al citar: "la dignidad de la persona remite a una cualidad exclusiva, indefinida y simple del ser humano, que designa su superioridad frente al resto de los seres con independencia del modo de comportarse". También Hervada (1991) resume el concepto de dignidad humana de la siguiente manera: "es la perfección o intensidad del ser que corresponde a la naturaleza humana y que se predica de la persona, en cuanto ésta es la realización existencial de la naturaleza humana". La dignidad es un atributo que tiene la persona por el solo hecho de ser. Sin embargo, Andorno (2019) recuerda que tanto en Derecho como en 
Filosofía el concepto de dignidad presenta problemas al ser definido precisamente por la concepción filosófica en que debemos fundarnos, tal vez sea por eso que al definir dignidad nos centremos solo en la condición de ser humano, independiente de sus circunstancias.

Tal como lo dice nuevamente Spaemann (1988) la dignidad es un término que se aplica al hombre para señalar una particular manera de ser o cualidad de ser, para sostener que es persona y no individuo, ser persona de esta manera no es una cualidad añadida, es su realidad misma, su existencia concreta.

De acuerdo con Sánchez Cámara (2019) "la idea de persona entraña la de la posesión de una especial dignidad", independientemente de que pueda perder determinadas cualidades como consecuencia de una enfermedad $o$ de un terrible sufrimiento, seguirá siendo un ser con sentido de trascendencia. De manera que la dignidad no dependerá de las cualidades físicas, mentales o psicológicas que posee la persona, sino que por el solo hecho de ser persona, todas poseen igual dignidad.

Desde el punto de vista ontológico y luego de siglos de pensamiento llegando hasta el pensamiento cristiano, encontramos en Kant (1992) el concepto de que la dignidad también remite a la idea de persona como un fin en sí misma y no un medio, en su Fundamentación de la Metafísica de las Costumbres citaba que:

Las personas no son meros fines subjetivos, cuya existencia, como efecto de nuestra acción, tiene un valor para nosotros, sino que son fines objetivos, esto es, seres cuya existencia en sí misma es un fin, y un fin tal que en su lugar no puede ponerse ningún otro fin para el cual debieran ellos servir como medios (...) los seres racionales se llaman personas porque su naturaleza los distingue ya como fines en sí mismos, esto es, como algo que no puede ser usado meramente como medio, y por tanto limita en ese sentido todo capricho y es objeto de respeto.

De tal forma que podemos afirmar con Kant que toda persona siempre es un fin en sí mismo y nunca un medio.

Es interesante resaltar que al hablar de persona humana debemos entenderla como un todo de cuerpo y alma y obviamente respetar también su corporalidad, pero más allá de ello, una persona tiene un espacio-tiempo histórico y muchas dimensiones y en todas y cada una de ellas debemos respetar su dignidad, recordemos que como seres finitos y existentes en el tiempo también tenemos expectativas de vida, también tenemos potencialidades y si no las desarrollamos no por eso dejamos de ser dignos.

Veamos ahora el segundo término: ¿a quiénes vamos a llamar enfermos terminales? Por definición enfermedad terminal es aquella enfermedad crónica y progresiva cuya expectativa de vida no va más allá de los 6 meses y cuya patología subyacente no es capaz de revertir con los tratamientos que actualmente se pueden ofrecer. Desde el punto de vista médico, sin embargo, nos basamos en estudios grandes, epidemiológicos, mundiales o regionales y estos muchas veces no son aplicables a nuestra realidad, pero, al final resultan ser por lo menos una guía para adecuar nuestro actuar frente a este tipo de pacientes. La constante en todos estos casos, independiente de la causa, es que son situaciones finales, recordemos que en todo caso el concepto que debiéramos manejar es el de proceso de irreversibilidad a corto tiempo.

\section{La dignidad humana amenazada en situaciones terminales}

En los párrafos anteriores hemos mostrado la íntima relación que existe entre el principio de dignidad humana $y$ el de persona. A 
continuación, trataremos brevemente del tema de la dignidad humana en situaciones terminales.

Sánchez Cámara (2019) se hace dos preguntas ante este tema y en relación con el sentido del dolor: ¿Puede el sufrimiento anular la dignidad de la vida? ¿Es indigna una vida extremadamente sufriente? Si reconocemos que el dolor es un concepto complejo y que del dolor también podemos sacar experiencias, obviamente la respuesta es negativa para ambas preguntas, sin olvidar que los creyentes que vivimos de cara a Dios "ofrecemos" el dolor para la salvación de uno y de los otros.

Las situaciones terminales con o sin dolor, en distintos pacientes son a su vez diferentes, pero hay que recordar que son situaciones en las que debemos medir riesgos, debemos controlar recursos, debemos administrar las limitaciones de la medicina y la limitación del ser humano ante la muerte. Llegados a este punto debemos tener en cuenta dos cosas: la persona en situación de enfermedad terminal es acreedora al mismo respeto a su dignidad que cualquier otra persona en cualquier otra circunstancia; y, que las condiciones "indignas" en que estas personas se encuentran no deben confundirse con la pérdida de la dignidad personal en casos terminales como lo refiere Masiá (2007), en los que la patología es letal, dejando que la enfermedad siga su curso no pretendiendo alargar la vida en condiciones más dolorosas o a veces hasta humillantes, teniendo cuidado de ofrecerle todos los medios a nuestro alcance para que tenga una buena calidad de vida hacia el final.

La Iglesia Católica, frente a los que afirman que la mayor parte de las veces las situaciones en las que se encuentran los enfermos terminales son indignas, defiende la postura de que la indignidad puede evitarse recurriendo a las soluciones adecuadas, sin necesidad de acabar con la propia vida. En "Eutanasia, iproblema católico o problema de todos?", Ciccone (1991) argumenta que no hay razón suficiente que permita afirmar sin más que es indigna la muerte que sea precedida y acompañada de sufrimiento.

Kant (1992), a propósito de algunos ejemplos de deberes, se refiere al suicidio en casos de "desgracias lindantes con la desesperación" y especifica que tal idea no es compatible con la naturaleza ni con la idea de la "humanidad como fin en sí":

$\mathrm{Si}$, para escapar a una situación dolorosa, se destruye él a sí mismo, hace uso de una persona como mero medio para conservar una situación tolerable hasta el fin de la vida. Mas el hombre no es una cosa; no es, pues, algo que pueda usarse como simple medio; debe ser considerado, en todas las acciones, como fin en sí. No puedo, pues, disponer del hombre, en mi persona, para mutilarle, estropearle, matarle.

En el mundo de hoy han aumentado mucho las "necesidades tecnológicas" en el área médica, así como cada vez más, tenemos protocolos de atención que nos dicen que hacer y cómo hacerlo en situaciones de enfermedad terminal. $Y$ aquí es preciso detenerse un poco porque desde el punto de vista médico, tan importante como saber qué hacer en determinadas circunstancias es saber qué no debemos hacer. Existen criterios para definir una urgencia, criterios para definir una emergencia, criterios para definir pacientes terminales, criterios para ingresar a $\mathrm{UCl}$, criterios para muerte cerebral, etc. Pero iqué sucede con nosotros como docentes?, a los alumnos les enseñamos muchas veces que la muerte tiene que ser vencida, que no es posible perder un paciente, que estamos listos para todo y olvidamos que se trata con más respeto la dignidad del enfermo si sabemos hacer nuestro trabajo. Debemos saber que un paciente terminal no ingresa a $\mathrm{UCl}$, que a un paciente terminal se le cuida en una unidad de Cuidados Paliativos o en casa, que si no se le puede curar, 
se le alivia, se le da una buena calidad de vida, se prioriza el cómo debe morir y nunca el cuándo debe morir. Al respecto Chávez (1979) aclara: "la etapa terminal ha existido siempre, pero no así el problema de conciencia que suscita. Ese problema es de nuestro tiempo, fruto de los avances recientes de la medicina y de los recursos técnicos de los que hoy disponemos y que permiten sostener la vida del enfermo por días, por semanas o por meses, en ocasiones por años, casi a solicitud".

Hoy en día también es importante estudiar y plantearnos el proceso de morir sobre todo en pacientes con enfermedades terminales porque precisamente en eso estriba el dilema, no nos enseñan a asumir la muerte como un proceso, no estamos acostumbrados a hablarlo, menos a saber cómo se acompaña a otro en este proceso. Los hermanos de San Juan de Dios en una de sus últimas publicaciones decían que "no hay enfermos incuidables, aunque haya enfermos incurables". También consideremos que fuimos criados en una sociedad donde asumir la muerte no es simple, a diferencia de otras culturas y religiones. Desde un punto de vista bioético sería interesante que aprendamos y enseñemos a percibir la muerte como un proceso y no como un momento, percibir que debemos vivir de cara a la muerte pues empezamos a morir el día que nacemos y de esta manera acompañaremos mejor a quien va a morir.

Precisamente con el advenimiento de la tecnología hoy tenemos ventiladores de última generación que respiran por uno y casi sin ruido, pero la misma vida que juramos proteger al mismo tiempo está amenazada, mantenemos a pacientes que sin esas ayudas habrían muerto hace mucho; pero, también es cierto que la conciencia de la autonomía del paciente ha empezado a tener cada vez un protagonismo mayor y muchas veces sin el verdadero sentido de la misma, pues el paciente hoy siente amenazada su autonomía y su dignidad por este modo "tecnologizado" de morir.
Los problemas éticos en torno a la vida y a la muerte en pacientes en situación terminal se centran en tres puntos: el rechazo de los recursos médicos exagerados (obstinación terapéutica), el rechazo de la aceleración irresponsable de la muerte (eutanasia) y el punto medio que sería el fomento de los Cuidados Paliativos. De acuerdo con Masiá (2007), lo inteligente es plantearse la siguiente pregunta: ¿Se respetó en esta decisión la autonomía y dignidad de la persona teniendo en cuenta también las repercusiones en otras personas? ¿Es este respeto la motivación básica que apoya la decisión última cualquiera que ésta sea? Sin embargo, creemos que hay que ir más allá y prestar atención a las diferencias radicales que existen entre culturas y religiones; que bien miradas no son tantas y tampoco tan radicales, porque donde existe un sentir religioso o trascendental el respeto a la dignidad de la persona, el respeto al momento de la muerte y el después, casi siempre es muy parecido; quienes solemos hacer las diferencias somos las personas no las comunidades.

Por su parte Masiá (2007), en la sección titulada "Pensar la terminalidad con ética preventiva", nos lleva a lo que en Estados Unidos se denomina "Death education" o educación para aprender a morir, asumir la muerte y acompañar a quien muere; la muerte en el sentido de replantear el sentido de la misma para poder aproximarnos al sufriente con otra óptica y enseñarle también otra perspectiva.

A pesar de todo lo que tecnológicamente hemos avanzado aun adolecemos de criterios claros y universales para tomar decisiones al final de la vida, y por eso el surgimiento de los Comités Hospitalarios de Ética y los Centros de Cuidados Paliativos. Lo que no debemos de olvidar es que dignidad de la persona ante la muerte y cuidados paliativos son el binomio de oro en el manejo que tendríamos que sugerir en pacientes en situación terminal. Sin embargo, en 
relación a morir con dignidad no olvidemos que muerte digna, primero, no significa eutanasia, y sí, escoger el cómo se debe morir, nunca el cuándo, porque aun despojándolo de su sentido religioso, solo se puede disponer de algo que uno obtuvo, compró o se dio, y la vida no es algo que nosotros nos dimos, la vida es un don, un regalo, independiente del ser creyente o no.

Un punto interesante para ser tocado brevemente es el del testamento vital (living well en habla inglesa) y Masiá (2007) al respecto es partidario de hacer tres recomendaciones: primero, que el paciente redacte por escrito sus últimas decisiones sobre el final de su vida; segundo, que a nivel de sociedad médica y civil se fomente el diálogo sobre estos temas para poder tener un acceso adecuado y equitativo a los servicios de salud y a las unidades de cuidados paliativos; y tercero, que se hable de estos temas al interior de la familia con el fin de educarnos mutuamente en el difícil tema de morir.

Está claro que necesitamos hacer concreto el significado de la dignidad humana ante la muerte pero como dice Bayés (2017):

El sufrimiento es consecuencia de una valoración personal y subjetiva de que algo amenaza o daña seriamente nuestra existencia o integridad, $o$ la de alguien $o$ algo que consideramos de importancia vital para nosotros $y$ de que no tenemos recursos para evitar o reparar ese daño, por lo que la muerte en ocasiones puede conllevar un sufrimiento ante la impotencia de que no podemos hacer nada por evitarla.

Si bien el valor de la vida es irrenunciable, una política asistencial adecuada y pertinente sería aquella que incluya un oportuno y claro manejo integral de los pacientes en situación terminal tanto en el hospital como en los domicilios particulares.

\section{La dignidad humana como obligación de preservar la propia vida}

Analizada ya en los párrafos anteriores la necesidad de cuidar la dignidad humana de los pacientes en situación terminal, abordaremos a continuación el estudio de la dignidad humana como obligación de preservar la vida.

Muchos autores han considerado que la dignidad humana en el morir es un valor que ante todo y como presupuesto implica la obligación de conservar la propia vida, en ese sentido, cualquier medio que acorte la propia vida o la de los demás entra dentro del concepto de eutanasia.

Para García (2012) la dignidad como constructo es superior al principio de autonomía. En este siglo en que a nivel asistencial la relación médico-paciente ha pasado a ser horizontal y dialogante, existe el concepto de que la autonomía del paciente está por encima del bien y del mal. La persona cree que en función de su autonomía puede decidir acerca de su vida porque es independiente, pero se olvida que la autonomía es muy cambiante y nuestra naturaleza será casi siempre dependiente (se es recién nacido, se tiene retardo mental, se está prostrado en cama, etc.) Mientras que siempre somos dignos por el mero hecho de existir, no es una cualidad que alguien nos da, la poseemos desde antes de nacer, es un valor absoluto y subyace a los principios en los que se basan los derechos humanos.

La autonomía es un principio valioso y está limitado precisamente por la dignidad; sin embargo, para las ideologías utilitaristas se puede justificar la moralidad de un acto autónomo por el hecho de que no hace daño a nadie, por ejemplo, el suicidio que solo le hace daño al suicida, olvidando que con este presupuesto está vulnerando a su vez la "inviolabilidad de la vida humana", por lo que 
no es lógico apelar a este principio para precisamente acabar con la propia autonomía. De acuerdo con García "la exageración de la autonomía acaba en una fractura psicopatológica del sujeto o como decía Bertrand Russell en la esquizofrenia del hombre moderno".

De manera que solo la dignidad es siempre un atributo esencial al ser humano y al mismo tiempo una limitante para su autonomía, así como un presupuesto que nos obliga necesariamente a conservar la vida de uno mismo y de los otros.

\section{Conclusiones}

- El enfermo en situación terminal es y será siempre digno porque el ser digno obedece simplemente a "ser", no a las circunstancias que lo rodean.

- El proceso de morir debe ser discutido, socializado y aceptado, para de esa manera poder ayudar a pacientes en estado terminal a tener verdaderamente una muerte digna.

- La eutanasia será siempre como tal un atentado a la vida a la dignidad humana y debemos aprender a hacerle frente con los medios que ahora tenemos y que se llaman Cuidados Paliativos.

- No es lícito bajo ningún punto de vista y evocando el principio de autonomía, tomar la propia vida.

\section{Bibliografía}

Andorno, R. (2019). la dignidad humana como principio biojurídico y como estándar moral de la relación médico-paciente. Consejo Superior de Investigaciones Científicas.
Aparisi Miralles, Á. (2013). El principio de la dignidad humana como fundamento de un bioderecho global. Cuadernos de Bioética.

Bayés, R. (2017). Sufrimiento en la enfermedad terminal. Revista Psicología de la Salud.

Chávez, I. (1979). Morir digno y decisión médica. México: Syntex.

Ciccone, L. (1991). Eutanasia, problema cattolico o problema di tutti? Roma: Città Nuova.

D'Agostino, F. (2002). La dignidad humana, tema bioético. En A. M. González, E. Postigo, \& S. Aulestiarte, Vivir y morir con dignidad (pág. 27).

Declaración Universal de los Derechos Humanos. (10 de diciembre de 1948). Organización de las Naciones Unidas. París.

García Cuadrado, A. M. (2012). Problemas constitucionales de la dignidad de la persona. Persona y Derecho.

García Sánchez, E. (2012). Dignidad y vulnerabilidad: propuesta bioética de compatibilidad. Valencia: Universidad Católica de Valencia.

Hervada, J. (1991). Los derechos inherentes a la dignidad de la. Humana lura.

Kant, I. (1992). Fundamentación de la metafísica de las costumbres. (M. García Morente, Trad.)

Masiá, J. (2007). Dignidad humana y situaciones terminales. Anales del Sistema Sanitario de Navarra.

Sánchez Cámara, I. (2019). El valor y la dignidad de la vida terminal. Cuadernos de Bioética.

Spaemann, R. (1988). Sobre el concepto de dignidad humana. Persona y Derecho. 